\title{
Synthesis, Structure, and Magnetic Properties of Rare- Earth Benzoborole Complexes
}

Dezhao Zhu, ${ }^{\dagger}$ Mengmeng Wang ${ }^{\ddagger}$, Lulu Guo ${ }^{\dagger}$, Wei Shi, ${ }^{*}, \mathrm{Jianfeng}^{\mathrm{L} i},{ }^{\dagger}$ and Chunming Cui*,

${ }^{\dagger}$ State Key Laboratory of Elemento-Organic Chemistry, Nankai University, Tianjin 300071, China

${ }^{\ddagger}$ Key Laboratory of Advanced Energy Materials Chemistry (MOE), College of Chemistry, Nankai University, Tianjin 300071, China

*Corresponding Author(s): Wei Shi, E-mail: shiwei@nankai.edu.cn; Chunming Cui, E-mail: cmcui@nankai.edu.cn. 


\section{Table of Contents}

1 General Experimental........................................................ S3

2 X-ray Diffraction Parameters and Data............................... S5

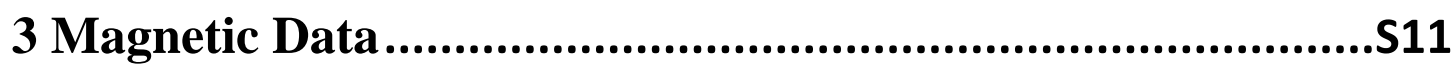

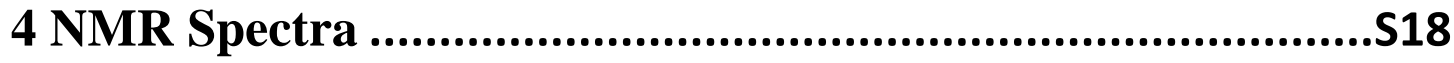

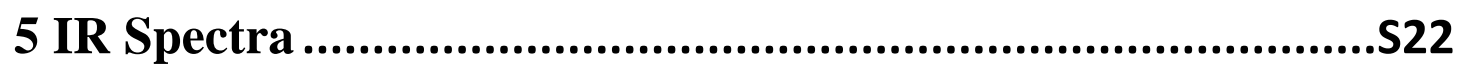

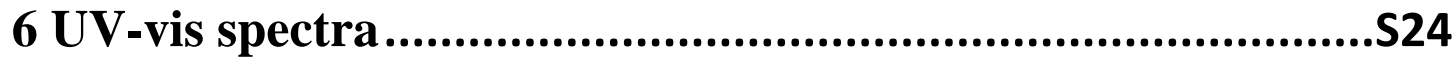

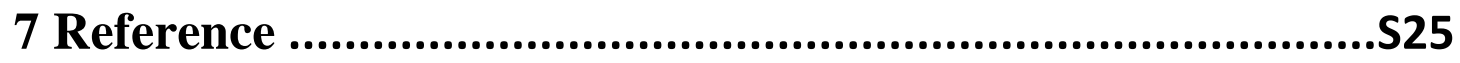




\section{General Experimental}

General Information. All manipulations involving air- and moisture-sensitive compounds were carried out under a dry argon atmosphere using standard Schlenk line and glovebox techniques. Glasswares were heat-dried and cooled down under vacuum prior to use. The solvents (THF, toluene, $\mathrm{Et}_{2} \mathrm{O}, \mathrm{DME}$ and $n$-hexane) were freshly distilled from sodium/benzophenone and degassed prior to use. $\mathrm{D}_{8}$-THF was obtained from J\&K Scientific and were dried by $\mathrm{Na} / \mathrm{K}$ alloy. All commercially available chemicals were employed as received if not stated otherwise. NMR spectra were recorded on Bruker AV-400FT $\left({ }^{1} \mathrm{H}\right.$ NMR at $400 \mathrm{MHz} ;{ }^{13} \mathrm{C}$ NMR at $101 \mathrm{MHz} ;{ }^{11} \mathrm{~B}$ NMR at $128 \mathrm{MHz} ;{ }^{7} \mathrm{Li}$ NMR at $156 \mathrm{MHz}$ ) NMR spectrometer. Elemental analysis was carried out on an Elemental Vario EL analyzer. Magnetic susceptibilities were performed on a Quantum Design SQUID MPMS VSM magnetometer. Diamagnetic corrections were carried out with Pascal's constants for all constituent atoms and sample holders. IR spectra were recorded in the range of $400-4000 \mathrm{~cm}^{-1}$ on a Bruker Alpha II spectrophotometer in glove box filled with Argon. UV-vis spectra were taken on a Shimadzu UV-2450 spectrometer. Benzoborole dilithuium (2H) and $\mathrm{K}_{2}(\mathrm{COT})$ were synthesized according to the published procedures. ${ }^{\mathrm{S} 1, \mathrm{~S} 2}$

Synthesis of benzoborole yttrium chloride: A solution of $\mathbf{2 H}(0.5 \mathrm{mmol}, 0.349 \mathrm{~g})$ in THF (15 $\mathrm{mL})$ was added dropwise to a stirred solution of anhydrous $\mathrm{YCl}_{3}(1 \mathrm{mmol}, 0.194 \mathrm{~g})$ in THF at $78^{\circ} \mathrm{C}$. The solution was allowed to warm slowly to room temperature and stirred for $5 \mathrm{~h}$. THF was removed in vacuo, and $30 \mathrm{~mL}$ of $\mathrm{Et}_{2} \mathrm{O}$ was added. The residuals were filtered and the filtrate was concentrated. Removal of all volatiles afforded red solid, which has been characterized by ${ }^{1} \mathrm{H},{ }^{11} \mathrm{~B}$ and ${ }^{7}$ Li NMR spectroscopies (Figures S15, S16 and S17).

Synthesis of 3Y: A solution of $\mathbf{2 H}(0.5 \mathrm{mmol}, 0.349 \mathrm{~g})$ in THF $(15 \mathrm{~mL})$ was added dropwise to a stirred solution of anhydrous $\mathrm{YCl}_{3}(1 \mathrm{mmol}, 0.194 \mathrm{~g})$ in $\mathrm{THF}$ at $-78{ }^{\circ} \mathrm{C}$. The solution was allowed to warm slowly to room temperature and stirred for $5 \mathrm{~h}$. The mixture was cool down to $78{ }^{\circ} \mathrm{C}$ and $10 \mathrm{~mL}$ THF solution of freshly prepared $\mathrm{K}_{2}(\mathrm{COT})(1 \mathrm{mmol})$ was added dropwise. The solution was allowed to warm slowly to room temperature and stirred overnight. Removal of volatiles afforded red solid. It was digested with $30 \mathrm{~mL}$ of $\mathrm{Et}_{2} \mathrm{O}$ and $\mathrm{LiCl}$ was removed by filtration. Removal of all volatiles, the residuals were dissolved in DME $(10 \mathrm{~mL})$ and stored at room temperature to yield $\mathbf{3 Y}$ as red crystals. Yield: $0.475 \mathrm{~g}$ (based on single crystals), 65\%. Mp: 94-97 
${ }^{\circ} \mathrm{C}$. Elemental analysis calcd (\%) for $\mathrm{C}_{38} \mathrm{H}_{58} \mathrm{BLiNO}_{6} \mathrm{Y}: \mathrm{C}, 62.39 ; \mathrm{H}, 7.99 ; \mathrm{N}, 1.91$. Found: $\mathrm{C}, 62.51$; H, 8.08; N, 1.88. ${ }^{1} \mathrm{H}$ NMR (400 MHz, D 8 -THF) $\delta 7.10(\mathrm{~d}, J=8 \mathrm{~Hz}, 1 \mathrm{H}, \mathrm{Ph}), 6.88(\mathrm{~d}, J=8 \mathrm{~Hz}, 2 \mathrm{H}$, Ph), 6.77 (d, $J=8 \mathrm{~Hz}, 1 \mathrm{H}, \mathrm{Ph}), 6.68$ (d, $J=8 \mathrm{~Hz}, 2 \mathrm{H}, \mathrm{Ph}), 6.20$ (t, $J=8 \mathrm{~Hz}, 1 \mathrm{H}, \mathrm{Ph}), 6.13$ (t, $J=8$ $\mathrm{Hz}, 1 \mathrm{H}, \mathrm{Ph}), 5.96$ (t, $J=8 \mathrm{~Hz}, 1 \mathrm{H}, \mathrm{Ph}), 5.84$ (s, 8H, COT), 4.98 (s, 1H, CHCCMe 3$), 4.40$ (s, 1H, $\mathrm{NH}), 3.42$ (s, 8H, CH $\left.\mathrm{CHME}_{2(\mathrm{DM})}\right), 3.26$ (s, 12H, $\left.\mathrm{CH}_{3(\mathrm{DME})}\right), 1.18$ (s, 9H, CMe $) .{ }^{13} \mathrm{C} \mathrm{NMR}\left(101 \mathrm{MHz}, \mathrm{D}_{8^{-}}\right.$ THF) $\delta 151.4,131.9,127.9,127.7,121.1,115.4,114.4,111.4,109.9,93.0\left(\mathrm{CHCCMe}_{3}\right), 92.3$ (COT), $71.7\left(\mathrm{CH}_{2(\mathrm{DME})}\right), 58.0\left(\mathrm{CH}_{3(\mathrm{DME})}\right), 33.7\left(\mathrm{CMe}_{3}\right), 33.5\left(\mathrm{CMe}_{3}\right)$, the resonances for the carbon atoms adjacent to the boron could not be observed. ${ }^{11} \mathrm{~B}$ NMR (128 MHz, $\mathrm{D}_{8}$-THF) $\delta$ 19.2. ${ }^{7} \mathrm{Li}$ NMR (156 MHz, D8-THF) $\delta$-1.0. IR v/cm ${ }^{-1}: 3406$ (w, N-H), 3039 (w), 2928 (w), 2823 (w), 1592 (s), 1495 (s), $1319(\mathrm{~s}), 1079(\mathrm{~s}), 839$ (s), $692(\mathrm{~s}), 485$ (w), $405(\mathrm{w})$.

Synthesis of 3Dy: Complex 3Dy was prepared similar to $3 \mathrm{Y}$ using anhydrous $\mathrm{DyCl}_{3}(1 \mathrm{mmol}$, $0.269 \mathrm{~g}$ ). Red crystals. Yield: $0.556 \mathrm{~g}$ (based on single crystals), 69\%. Mp: 111-114 ${ }^{\circ} \mathrm{C}$. Elemental analysis calcd (\%) for $\mathrm{C}_{38} \mathrm{H}_{58} \mathrm{BLiNO}_{6} \mathrm{Dy}$ : C, 56.69; H, 7.26; N, 1.74. Found: C, 56.76; H, 7.34; N, 1.68. IR v/cm $\mathrm{cm}^{-1}: 3406$ (w, N-H), 3035 (w), 2925 (w), 1588 (w), 1486 (s), 1253 (w), 1189 (w), 1075 (s), 1019 (s), $861(\mathrm{w}), 803(\mathrm{w}), 741$ (w), 690 (s), 482 (w), 432 (w).

Synthesis of 3Er: Complex $3 \mathbf{E r}$ was prepared similar to $3 \mathbf{Y}$ using anhydrous $\mathrm{ErCl}_{3}(1 \mathrm{mmol}$, $0.271 \mathrm{~g}$ ). Red crystals. Yield: $0.582 \mathrm{~g}$ (based on single crystals), $72 \%$. Mp: $140-143{ }^{\circ} \mathrm{C}$. Elemental analysis calcd (\%) for $\mathrm{C}_{38} \mathrm{H}_{58} \mathrm{BLiNO}_{6} \mathrm{Er}$ : C, 56.36; H, 7.22; N, 1.73. Found: C, 56.47; H, 7.31; N, 1.67. IR v/cm ${ }^{-1}: 3405$ (w, N-H), 3028 (w), 2923 (w), 1587 (s), 1482 (s), 1311 (w), 1075 (s), 861 (w), $689(\mathrm{~s}), 484(\mathrm{w})$. 


\section{X-ray Diffraction Parameters and Data}

The X-ray crystallographic data for compounds 3Y, 3Dy and 3Er were collected with a Rigaku Saturn 724 CCD diffractometer using graphite-monochromated Mo K $\alpha$ radiation $(\lambda=0.71073 \AA)$ at $113(2) \mathrm{K}$. The structures were solved with the Olex2 and refined with the ShelXL refinement package using Least Squares minimization. ${ }^{\text {S3-S5 }}$ Refinement was performed on $F^{2}$ anisotropically for all the non-hydrogen atoms by the full-matrix least-squares method. The hydrogen atoms were placed at the calculated positions and were included in the structure calculation without further refinement of the parameters. The Olex 2 program was utilized to draw the molecular structures. ${ }^{\mathrm{S}}$ Details of the crystallographic data and a summary of the intensity data collection parameters for 3Y, 3Dy and 3Er are listed in Table S1. Molecular structure of 3Y, 3Dy and 3Er are shown in Figures S1-S3. 
Table S1. Crystallographic Data for Compounds 3Y, 3Dy and 3Er.

\begin{tabular}{|l|l|l|l|}
\hline Complex & $\mathbf{3 Y}$ & 3Dy & 3Er \\
\hline CCDC No & 2051944 & 2078115 & 2051946 \\
\hline formula & $\mathrm{C}_{38} \mathrm{H}_{58} \mathrm{BYLiNO}_{6}$ & $\mathrm{C}_{38} \mathrm{H}_{58} \mathrm{BDyLiNO}_{6}$ & $\mathrm{C}_{38} \mathrm{H}_{58} \mathrm{BErLiNO}_{6}$ \\
\hline fw & 731.36 & 806.38 & 808.38 \\
\hline$T(\mathrm{~K})$ & 113.15 & 113.15 & 113.15 \\
\hline space group & $P-1$ & $P-1$ & $P-1$ \\
\hline$a(\AA)$ & $9.7335(19)$ & $9.7076(19)$ & $9.803(2)$ \\
\hline$b(\AA)$ & $12.650(3)$ & $12.689(3)$ & $12.559(3)$ \\
\hline$c(\AA)$ & $16.257(3)$ & $16.233(3)$ & $16.309(3)$ \\
\hline$\alpha($ deg. $)$ & $98.16(3)$ & $98.13(3)$ & $98.35(3)$ \\
\hline$\beta($ deg. $)$ & $94.61(3)$ & $94.02(3)$ & $95.76(3)$ \\
\hline$\gamma($ deg. $)$ & $96.47(3)$ & $96.71(3)$ & $96.60(3)$ \\
\hline$V\left(\AA^{3}\right)$ & $1959.2(7)$ & $1958.3(7)$ & $1959.4(7)$ \\
\hline$Z(\AA 3)$ & 2 & 2 & 2 \\
\hline$\left.d_{\text {calcd }}(\mathrm{g} / \mathrm{cm})^{3}\right)$ & 1.240 & 1.365 & 1.373 \\
\hline$F(000)$ & 776.0 & 830.0 & 834.0 \\
\hline GOF & 0.965 & 1.088 & 0.989 \\
\hline$R_{I}(I>2 \sigma(I))$ & 0.0550 & 0.0497 & 0.07345 \\
\hline$w R_{2}(\mathrm{all}$ data) & 0.1189 & 0.1081 & \\
\hline
\end{tabular}



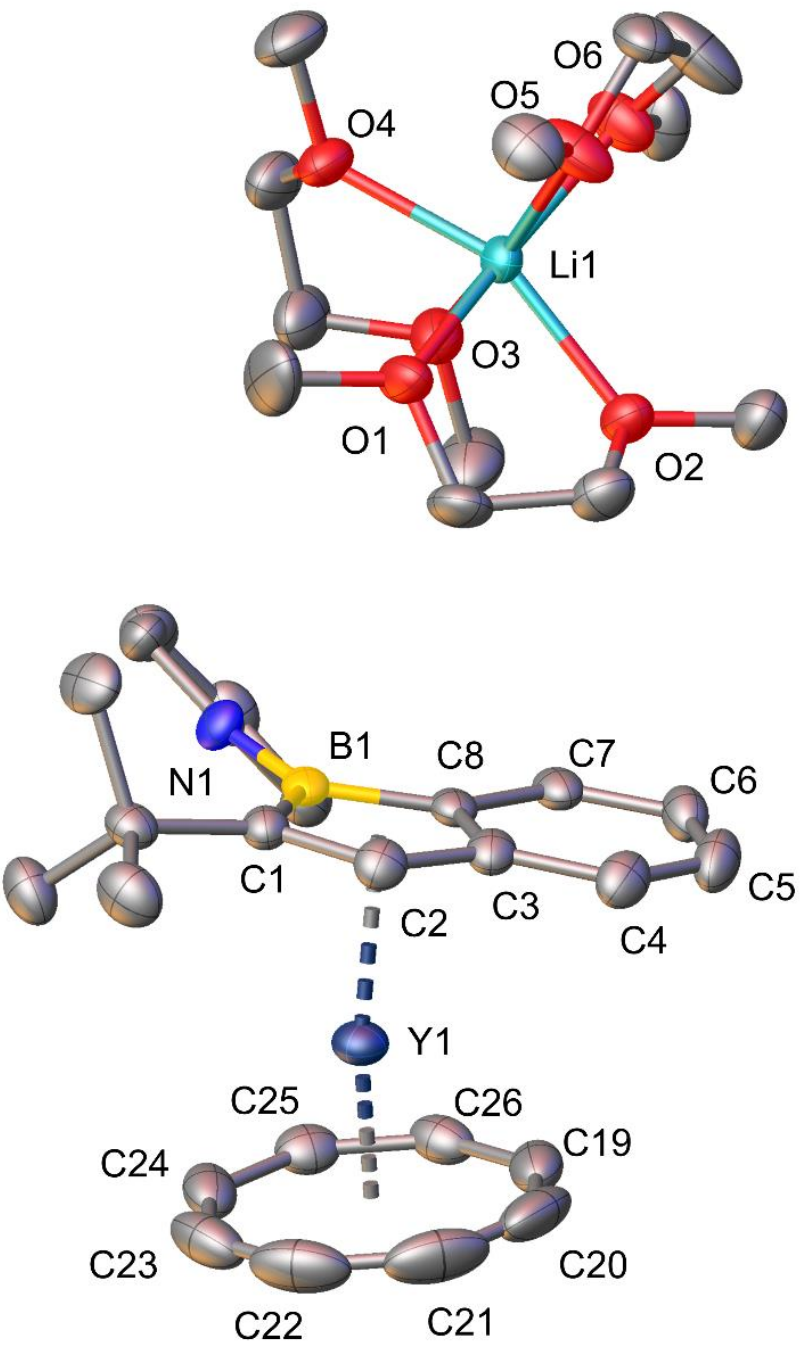

Figure S1. Molecular structure of $\mathbf{3 Y}$ with ellipsoids drawn at the $50 \%$ probability level. All hydrogen atoms are omitted for clarity. Selected bond lengths $(\AA)$ and angles (deg): B1-C1 1.526(4), C1-C2 1.441(4), C2-C3 1.410(4), C3-C8 1.465(4), C8-B1 1.541(4), B1-N1 1.491(4), Y1-C1 2.621(3), Y1-C2 2.524(3), Y1-C3 2.553(3), Y1-C8 2.653(3), Y1-B1 2.741(3), Y1-C19 2.528(3), Y1-C20 2.542(3), Y1-C21 2.553(3), Y1-C22 2.568(3), Y1-C23 2.573(3), Y1-C24 2.555(4), Y1C25 2.543(4), Y1-C26 2.522(4), C8-B1-N1 129.5(3), N1-B1-C1 125.8(3), C1-B1-C8 104.3(3), B1-C1-C2 108.0(2), C1-C2-C3 110.7(3), C2-C3-C8 110.1(3), C3-C8-B1 106.8(3). 


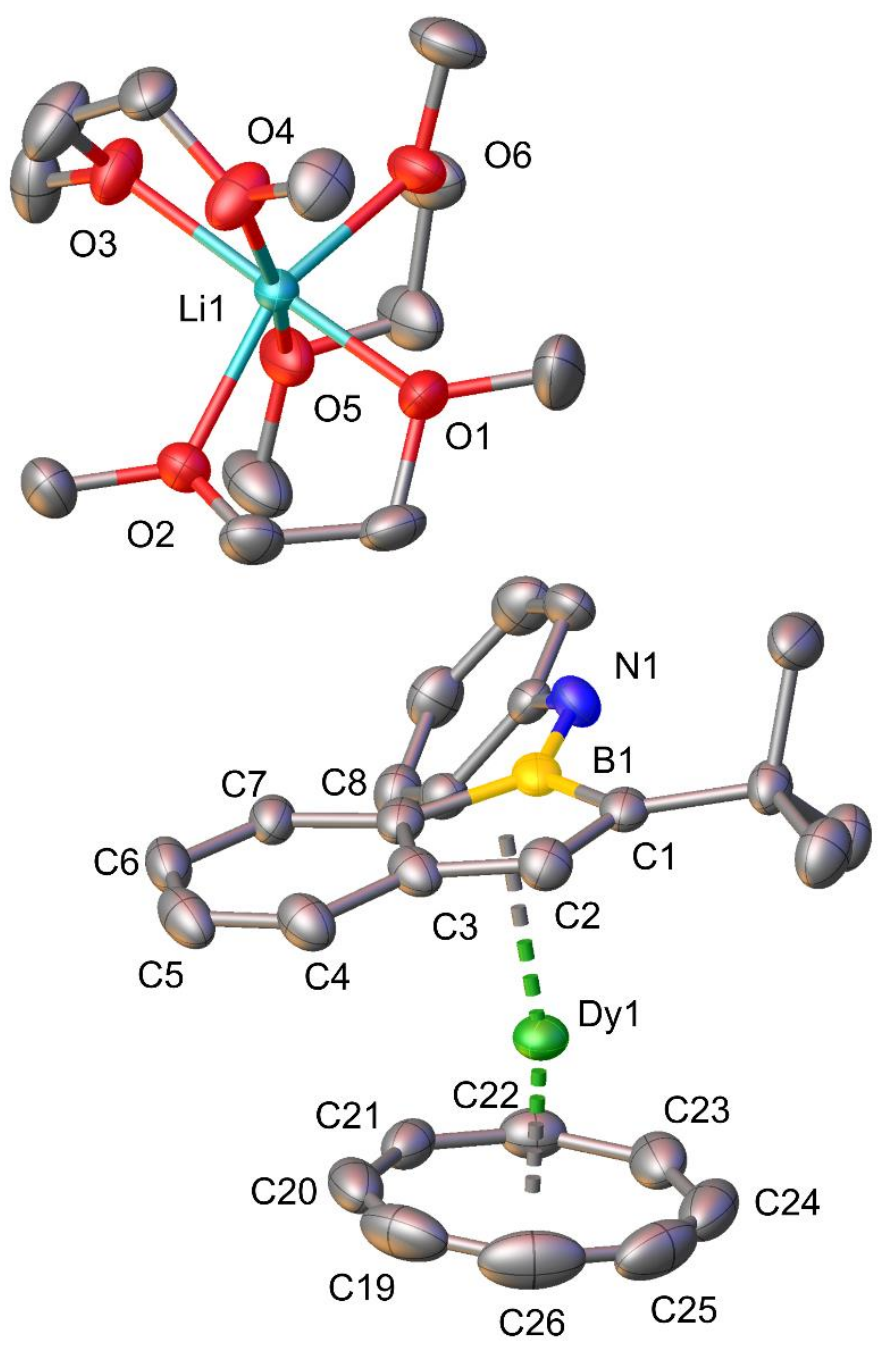

Figure S2. Molecular structure of 3Dy with ellipsoids drawn at the 50\% probability level. All hydrogen atoms are omitted for clarity. Selected bond lengths $(\AA)$ and angles (deg): B1-C1 1.530(6), C1-C2 1.435(6), C2-C3 1.411(6), C3-C8 1.468(6), C8-B1 1.558(6), B1-N1 1.490(6), Dy1-C1 2.626(4), Dy1-C2 2.535(4), Dy1-C3 2.569(4), Dy1-C8 2.677(4), Dy1-B1 2.759(4), Dy1-C19 2.538(5), Dy1-C20 2.534(5), Dy1-C21 2.539(5), Dy1-C22 2.555(5), Dy1-C23 2.576(5), Dy1-C24 2.587(5), Dy1-C25 2.560(5), Dy1-C26 2.548(5), C8-B1-N1 130.0(4), N1-B1-C1 125.8(4), C1B1-C8 104.0(4), B1-C1-C2 108.1(4), C1-C2-C3 111.3(4), C2-C3-C8 109.9(4), C3-C8-B1 106.6(4). 


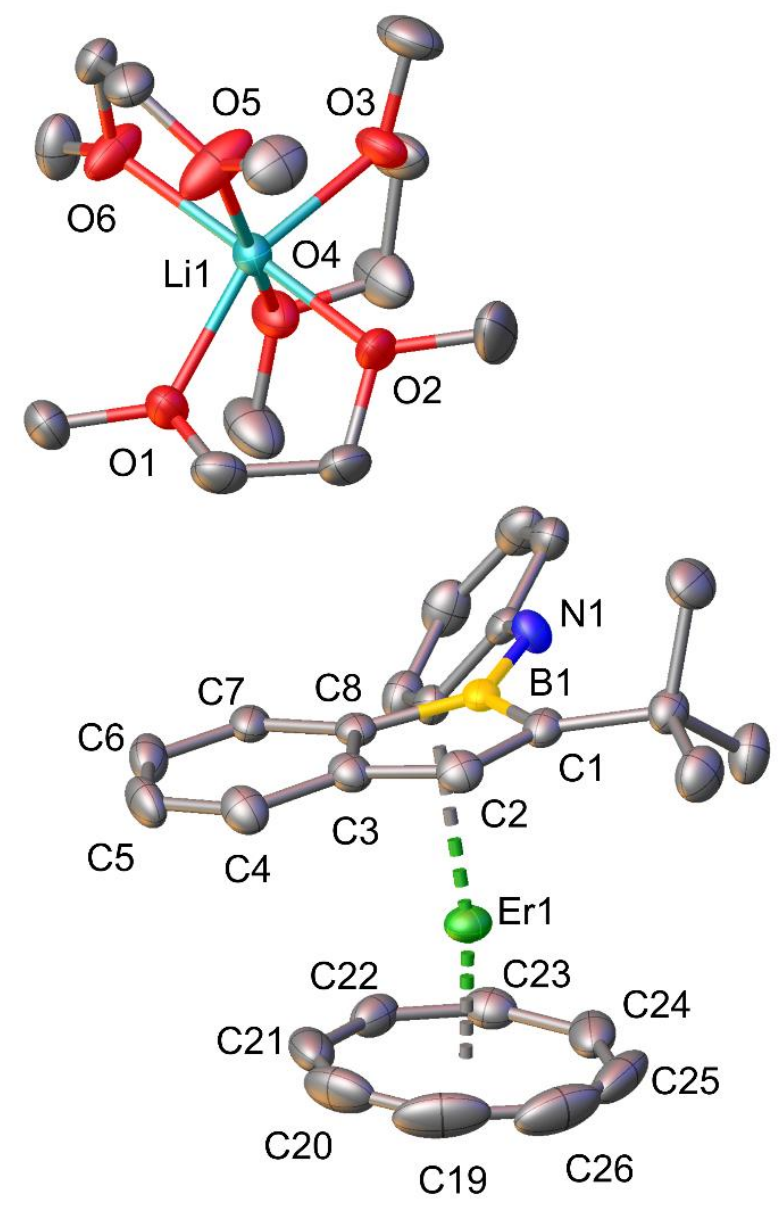

Figure S3. Molecular structure of 3Er with ellipsoids drawn at the 50\% probability level. All hydrogen atoms are omitted for clarity. Selected bond lengths $(\AA)$ and angles (deg): B1-C1 1.516(4), C1-C2 1.445(4), C2-C3 1.412(4), C3-C8 1.473(4), C8-B1 1.543(4), B1-N1 1.494(4), Er1-C1 2.593(3), Er1-C2 2.508(3), Er1-C3 2.540(3), Er1-C8 2.633(3), Er1-B1 2.716(3), Er1-C19 2.517(4), Er1-C20 2.491(4), Er1-C21 2.500(4), Er1-C22 2.505(3), Er1-C23 2.526(3), Er1-C24 2.546(3), Er1-C25 2.546(4), Er1-C26 2.531(4), C8-B1-N1 129.4(3), N1-B1-C1 125.4(3), C1B1-C8 104.9(3), B1-C1-C2 107.9(3), C1-C2-C3 110.8(3), C2-C3-C8 109.9(3), C3-C8-B1 106.4(2). 
Table S2. Selected Distances ( $\AA$ ) and Dihedral Angles (deg) for 3Y, 3Dy and 3Er (3RE).

\begin{tabular}{|c|c|c|c|}
\hline Complex & $3 \mathbf{Y}$ & 3Dy & $3 \mathbf{E r}$ \\
\hline $\mathrm{RE} \cdots \mathrm{RE}^{a}$ & 7.911 & 7.841 & 7.993 \\
\hline $\mathrm{RE}-\mathrm{C}(\mathrm{COT})^{b}$ & 2.548 & 2.555 & 2.520 \\
\hline $\mathrm{RE}-\mathrm{C}(\mathrm{Bo})^{b}$ & 2.588 & 2.602 & 2.569 \\
\hline RE-B & $2.741(3)$ & $2.759(4)$ & $2.716(3)$ \\
\hline $\mathrm{B}-\mathrm{N}$ & $1.491(4)$ & $1.490(6)$ & 1.494(4) \\
\hline $\mathrm{RE}-$ centroid $(\mathrm{COT})^{c}$ & 1.770 & 1.779 & 1.732 \\
\hline $\mathrm{RE}$-centroid $(\mathrm{Bo})^{c}$ & 2.299 & 2.314 & 2.275 \\
\hline Dihedral angle & 12.40 & 13.64 & 11.80 \\
\hline Angle of Bo cent-RE-COT ${ }_{\text {cent }}{ }^{d}$ & 168.74 & 167.24 & 169.34 \\
\hline
\end{tabular}

Bo represents benzoborole ring. ${ }^{a}$ Nearest-neighbor distance. ${ }^{b}$ Average value. ${ }^{c}$ Distance from ring centroid to metal. ${ }^{d}$ Angle of metal and two ring centroids. 


\section{Magnetic Data}

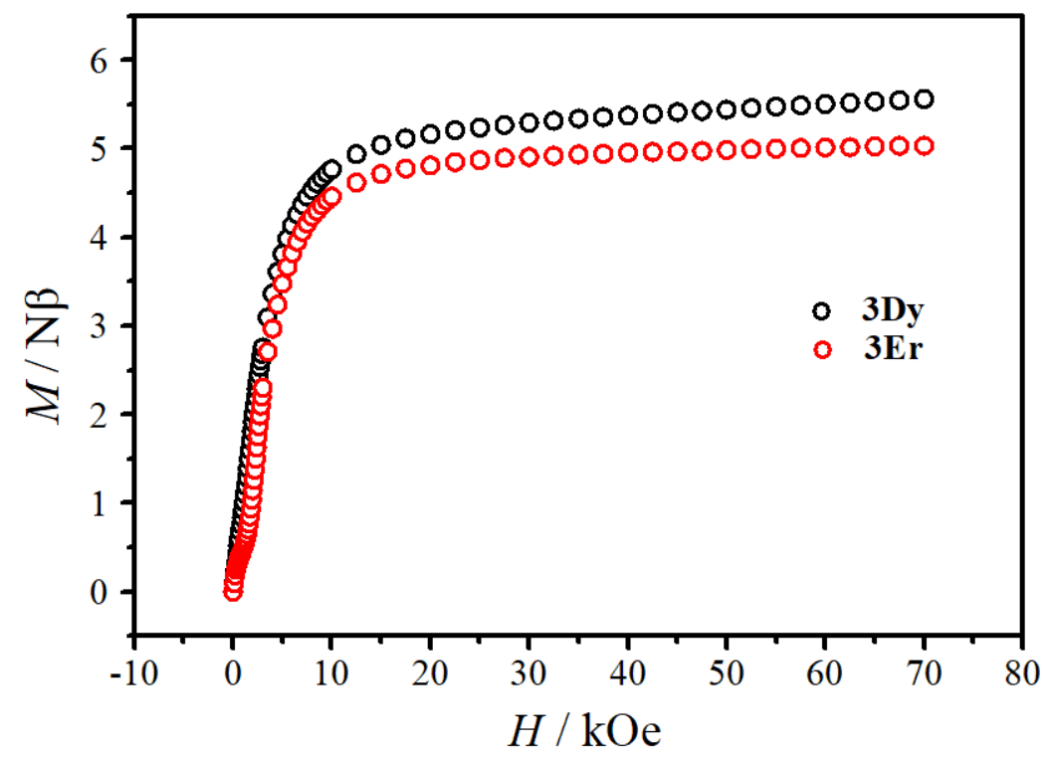

Figure S4. The field dependence of magnetization for 3Dy and 3Er at $2 \mathrm{~K}$.
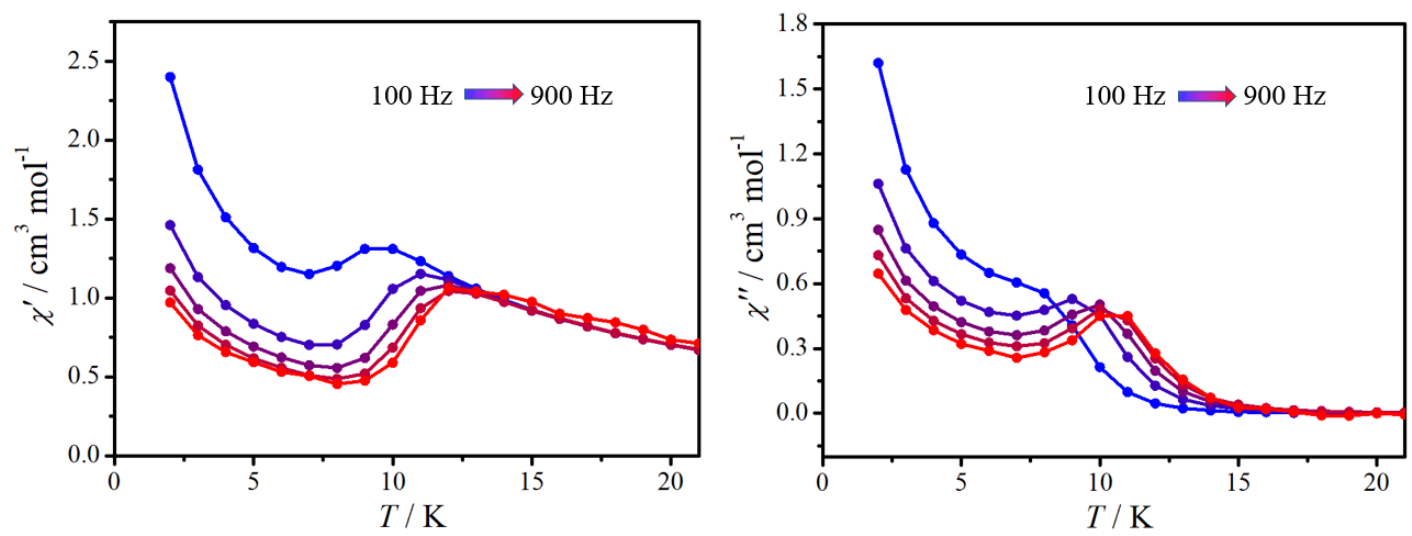

Figure S5. Temperature dependence of the (left) in-phase $\left(\chi^{\prime}\right)$ and (right) out-of-phase $\left(\chi^{\prime \prime}\right)$ of ac susceptibilities for $\mathbf{3 D y}$ at variable frequencies. 

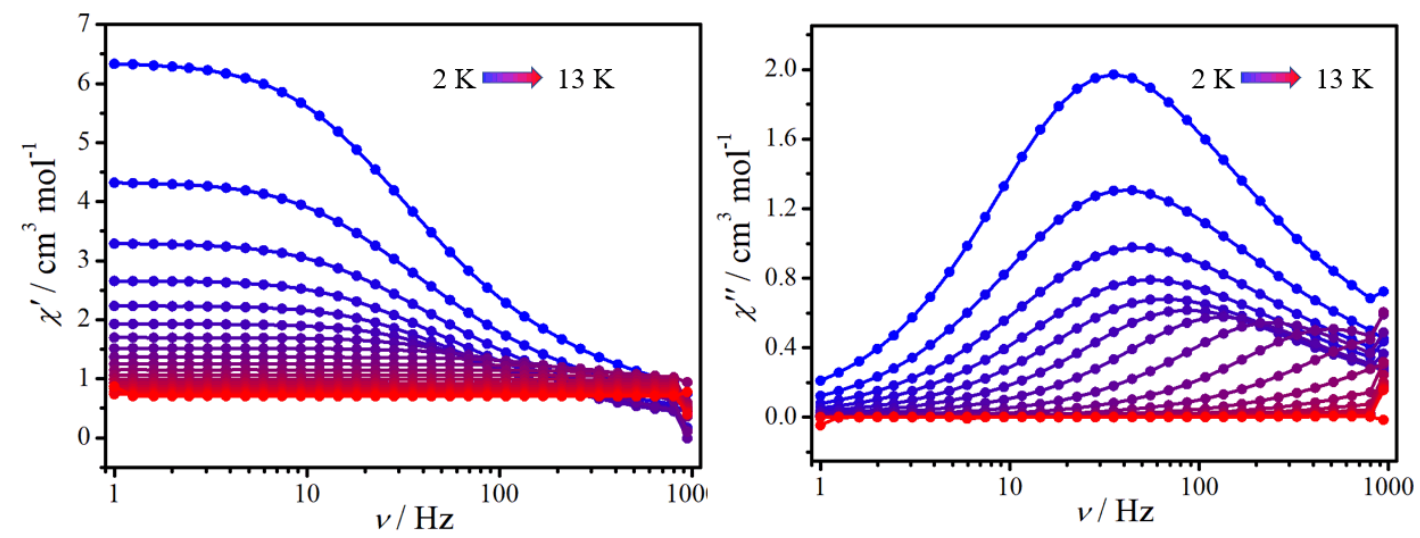

Figure S6. Frequency dependence of the (left) $\chi^{\prime}$ and (right) $\chi^{\prime \prime}$ of ac susceptibilities for 3Dy under zero dc field.

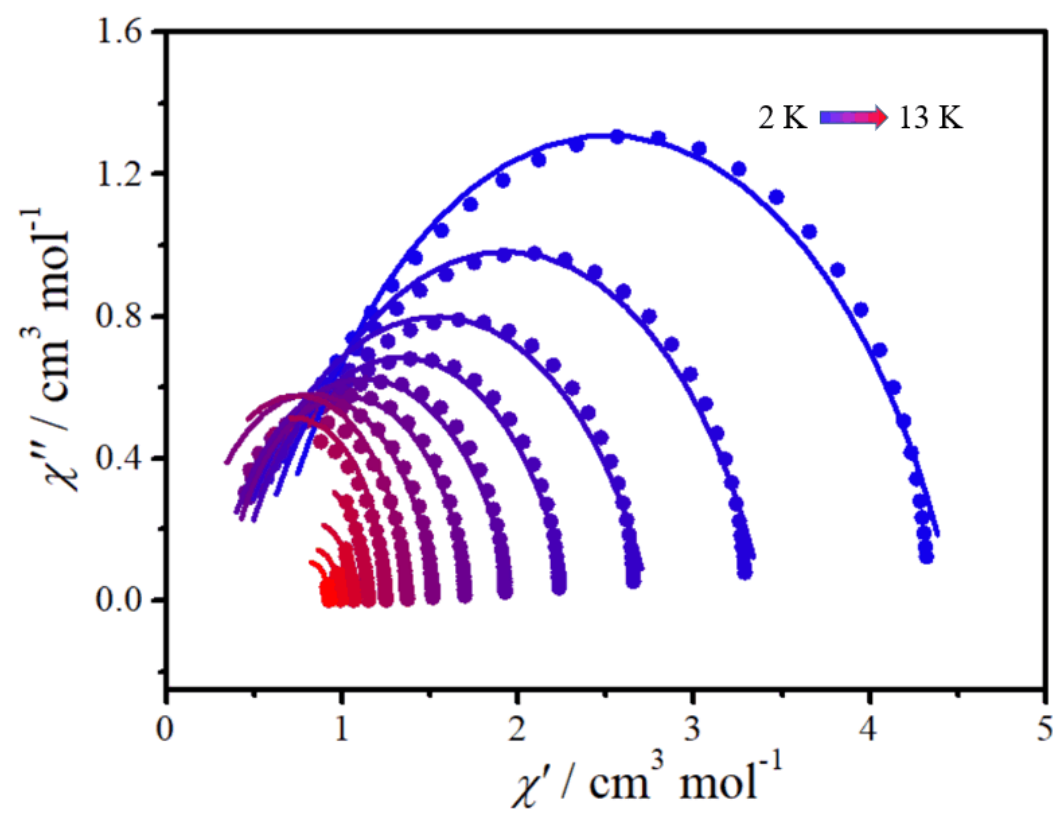

Figure S7. The Cole-Cole plots for 3Dy. The solid lines are fitting results by using the generalized Debye models. 


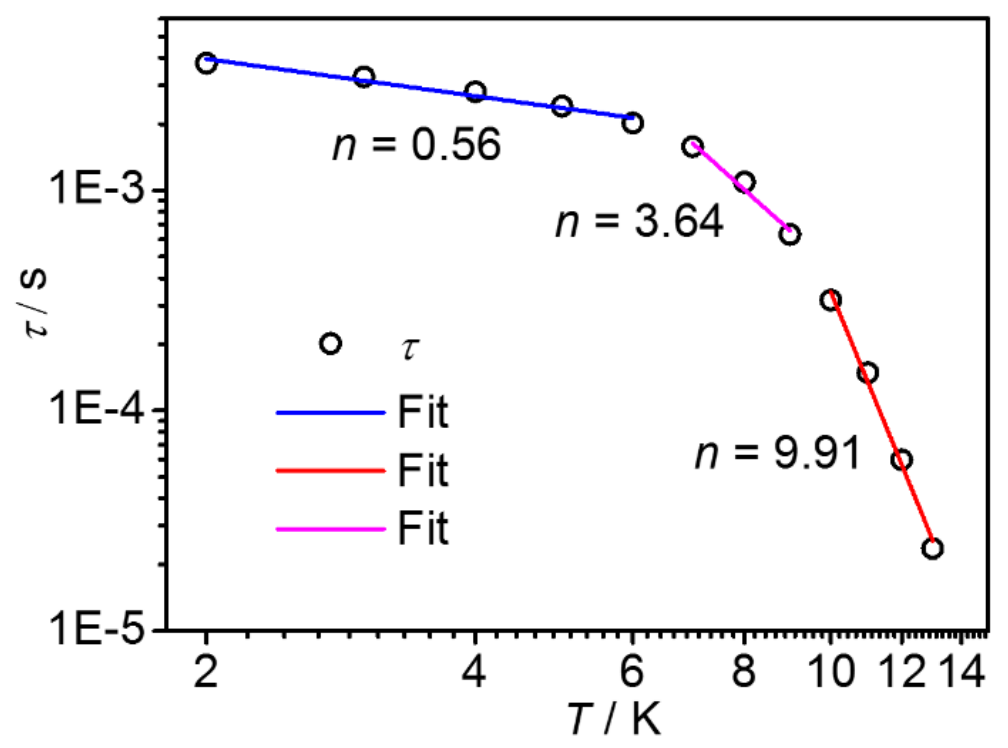

Figure S8. The $\tau$ versus $T^{\mathrm{n}}$ plot shown in $\log -\log$ scale for 3Dy.

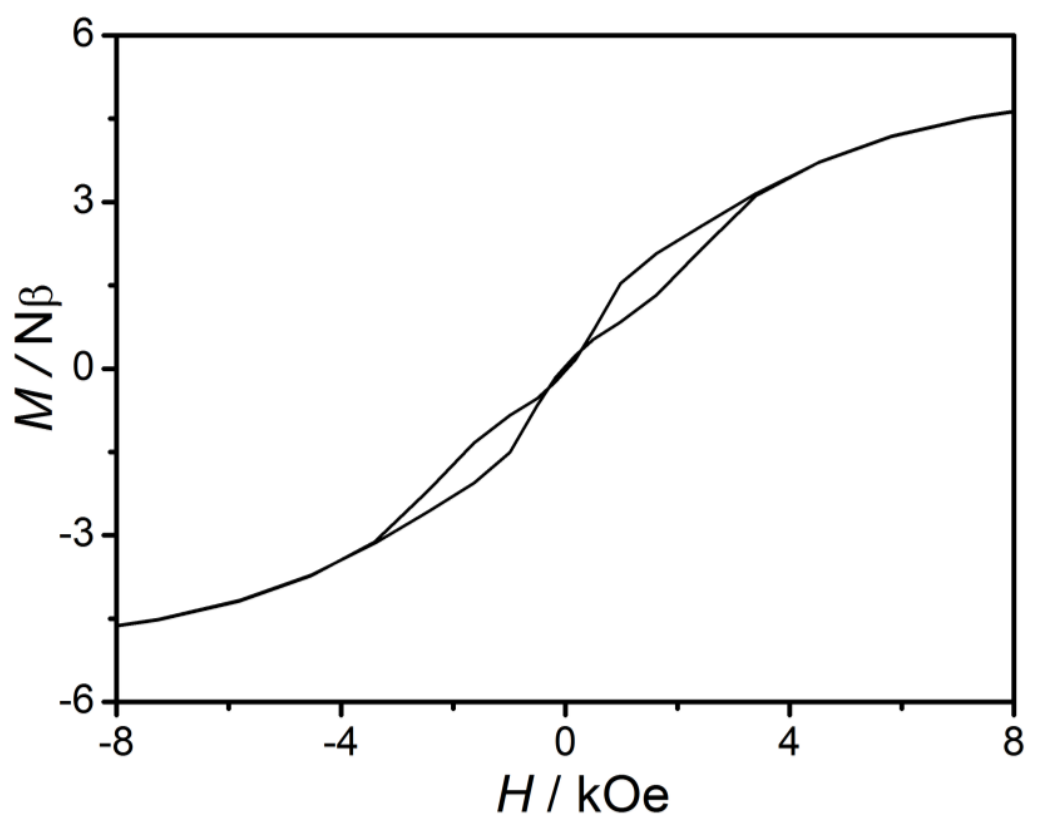

Figure S9. Magnetization hysteresis at $2 \mathrm{~K}$ for 3Dy. 

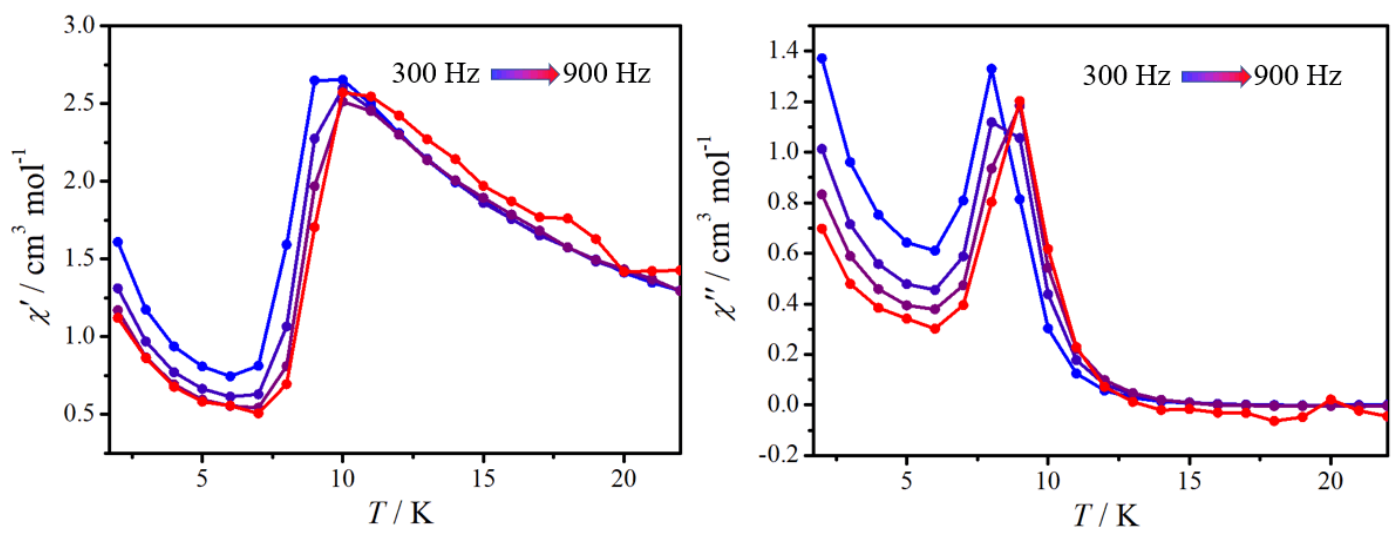

Figure S10. Temperature dependence of the (left) $\chi^{\prime}$ and (right) $\chi^{\prime \prime}$ of ac susceptibilities for 3Er under variable frequencies.
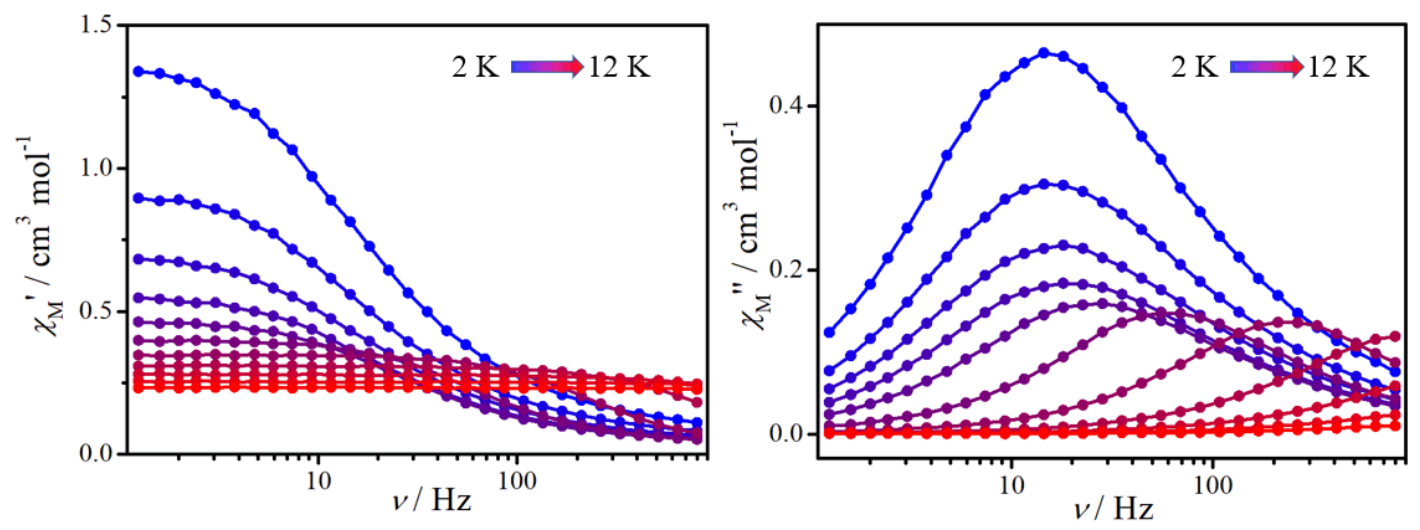

Figure S11. Frequency dependence of the (left) $\chi^{\prime}$ and (right) $\chi^{\prime \prime}$ of ac susceptibilities for 3Er under zero dc field. 


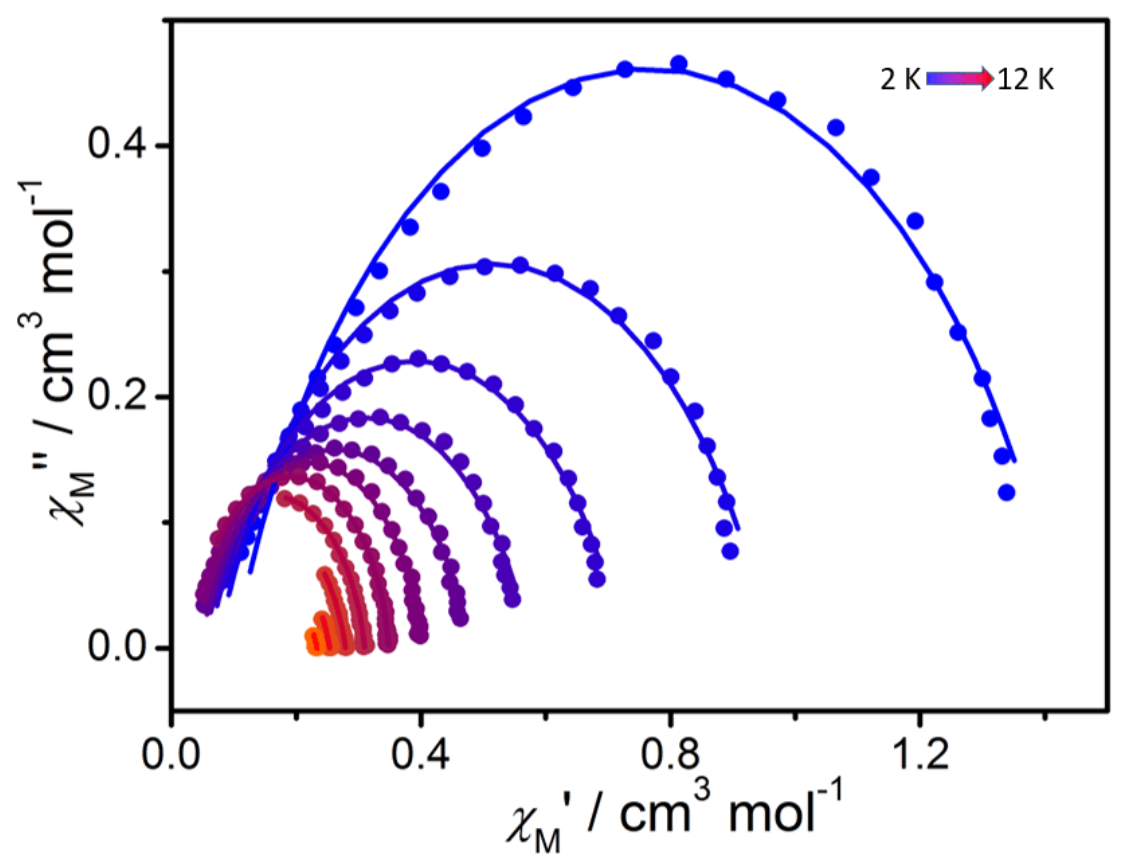

Figure S12. The Cole-Cole plots for 3Er. The solid lines are fitting results by using the generalized Debye models.

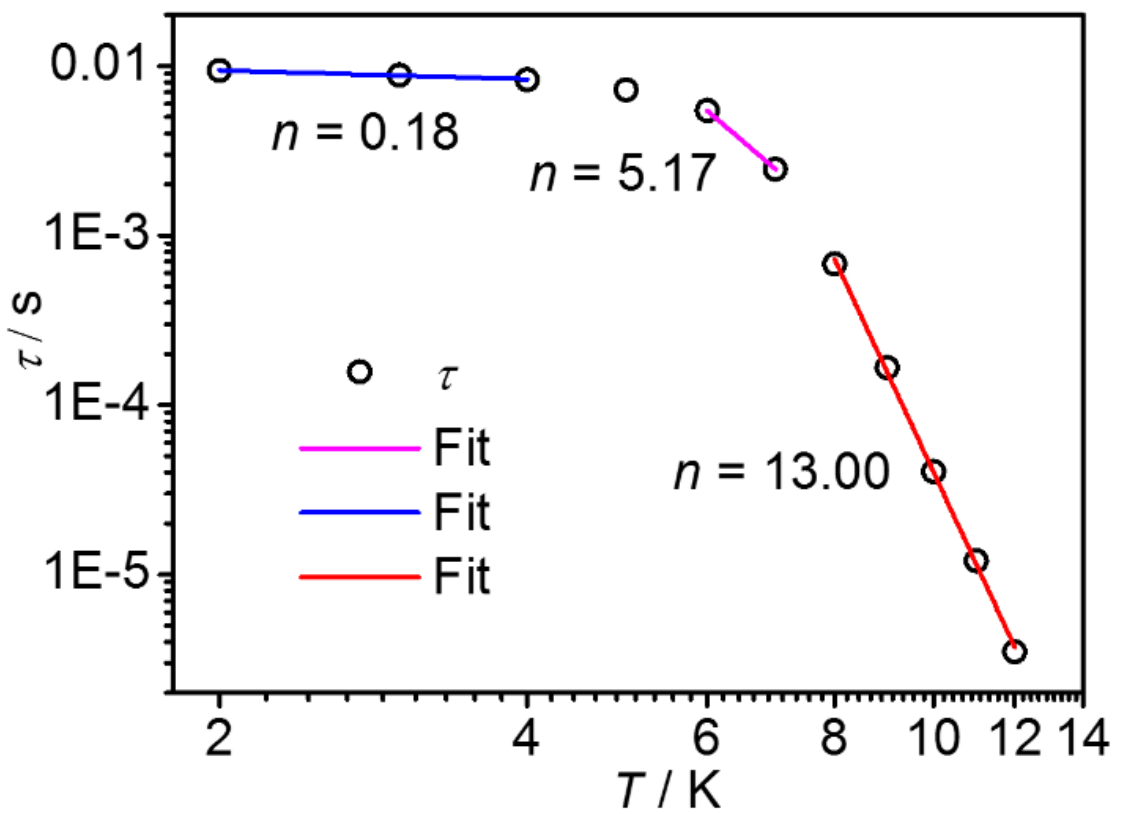

Figure S13. The $\tau$ versus $T^{\mathrm{n}}$ plot shown in log-log scale for 3Er. 


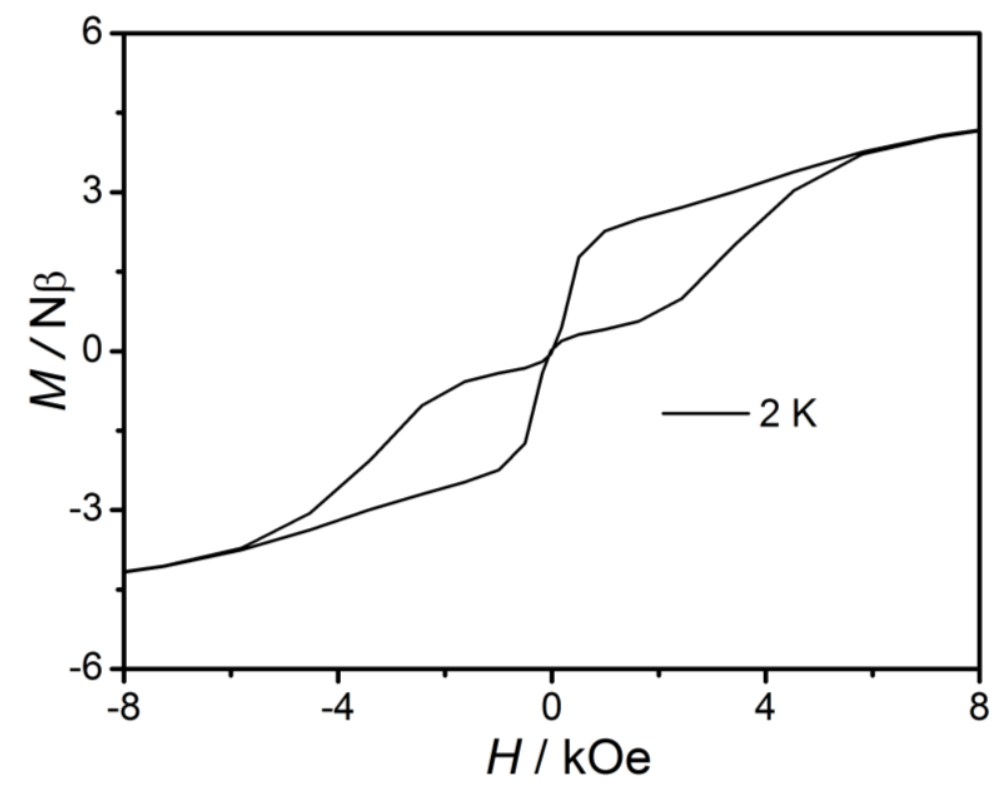

Figure S14. Magnetization hysteresis at $2 \mathrm{~K}$ for 3Er.

Table S3. Debye model fitting parameters for 3Dy.

\begin{tabular}{|c|c|c|c|c|c|}
\hline$T / \mathrm{K}$ & $\chi_{\mathrm{s}} / \mathrm{cm}^{3} \mathrm{~mol}^{-1}$ & $\chi_{\mathrm{T}} / \mathrm{cm}^{3} \mathrm{~mol}^{-1}$ & $\alpha$ & $\tau / \mathrm{s}$ & Residual \\
\hline 2 & 0.72926 & 6.55921 & 0.00378 & 0.24121 & 0.27516 \\
\hline 3 & 0.57163 & 4.46579 & 0.00327 & 0.24482 & 0.12402 \\
\hline 4 & 0.48569 & 3.38799 & 0.0028 & 0.24137 & 0.0678 \\
\hline 5 & 0.43445 & 2.72877 & 0.00241 & 0.22686 & 0.04481 \\
\hline 6 & 0.40023 & 2.28153 & 0.00202 & 0.19754 & 0.03124 \\
\hline 7 & 0.37135 & 1.95624 & 0.00158 & 0.1547 & 0.01938 \\
\hline 8 & 0.33843 & 1.71109 & 0.00109 & 0.10823 & 0.0088 \\
\hline 9 & 0.3064 & 1.52062 & $6.29695 \mathrm{E}-4$ & 0.0669 & 0.00246 \\
\hline 10 & 0.27896 & 1.3702 & $3.17545 \mathrm{E}-4$ & 0.04298 & $4.03717 \mathrm{E}-4$ \\
\hline 11 & 0.2494 & 1.24889 & $1.48858 \mathrm{E}-4$ & 0.04448 & $1.40043 \mathrm{E}-4$ \\
\hline 12 & 0.13313 & 1.1491 & $5.99567 \mathrm{E}-5$ & 0.07928 & $1.52507 \mathrm{E}-4$ \\
\hline 13 & $6.56785 \mathrm{E}-14$ & 1.06381 & $2.37121 \mathrm{E}-5$ & 0.11173 & $1.42786 \mathrm{E}-4$ \\
\hline
\end{tabular}


Table S4. Debye model fitting parameters for 3Er.

\begin{tabular}{cccccc}
\hline$T / \mathrm{K}$ & $\chi_{\mathrm{S} / \mathrm{cm}^{3} \mathrm{~mol}^{-1}}$ & $\chi_{\mathrm{T}} / \mathrm{cm}^{3} \mathrm{~mol}^{-1}$ & $\alpha$ & $\tau / \mathrm{s}$ & Residual \\
\hline 2 & 0.10022 & 1.42752 & 0.0094 & 0.22641 & 0.00759 \\
3 & 0.07376 & 0.95632 & 0.00884 & 0.22806 & 0.0042 \\
4 & 0.0581 & 0.72175 & 0.00831 & 0.23099 & 0.00214 \\
5 & 0.05049 & 0.57411 & 0.00722 & 0.2207 & 0.00155 \\
6 & 0.0466 & 0.4778 & 0.00546 & 0.19355 & 0.00107 \\
7 & 0.04222 & 0.4021 & 0.00246 & 0.12386 & $4.08937 \mathrm{E}-4$ \\
8 & 0.03443 & 0.3483 & $6.77863 \mathrm{E}-4$ & 0.08657 & $8.38242 \mathrm{E}-5$ \\
9 & 0.01711 & 0.30986 & $1.65629 \mathrm{E}-4$ & 0.12033 & $6.71403 \mathrm{E}-5$ \\
10 & $1.12678 \mathrm{E}-15$ & 0.27922 & $4.03417 \mathrm{E}-5$ & 0.17774 & $5.30066 \mathrm{E}-5$ \\
11 & $1.69427 \mathrm{E}-15$ & 0.25389 & $1.20389 \mathrm{E}-5$ & 0.21529 & $4.95601 \mathrm{E}-5$ \\
\hline 12 & $2.89175 \mathrm{E}-15$ & 0.23351 & $3.4887 \mathrm{E}-6$ & 0.26913 & $3.66006 \mathrm{E}-5$ \\
\hline
\end{tabular}

Table S5. Comparison of the key structural information and energy barriers of $\left(\mathrm{Cp}^{*}\right) \operatorname{Er}(\mathrm{COT})$, (Dsp)Er(COT), [( $\left.\left.\mathrm{C}_{5} \mathrm{H}_{5} \mathrm{BR}\right) \operatorname{Dy}(\mathrm{COT})\right]$, 3Dy and 3Er.

\begin{tabular}{|c|c|c|c|c|c|c|}
\hline & $\left(\mathrm{Cp}^{*}\right) \operatorname{Er}(\mathrm{COT})$ & (Dsp)Er(COT) & $3 E r$ & & {$\left[\left(\mathrm{C}_{5} \mathrm{H}_{5} \mathrm{BR}\right) \mathrm{Dy}(\mathrm{COT})\right]$} & 3Dy \\
\hline $\begin{array}{l}\text { Er-centroid } \\
(\mathrm{COT})(\AA)\end{array}$ & 1.661 & 1.686 & 1.732 & $\begin{array}{c}\text { Dy-centroid } \\
(\mathrm{COT})(\AA)\end{array}$ & $1.711-1.718$ & 1.779 \\
\hline $\begin{array}{l}\text { Er-centroid } \\
\text { (Five- } \\
\text { membered } \\
\text { ring) }(\AA)\end{array}$ & 2.264 & 2.321 & 2.275 & $\begin{array}{c}\text { Dy-centroid } \\
(\mathrm{BZ})(\AA)\end{array}$ & $2.277-2.307$ & 2.314 \\
\hline $\begin{array}{l}\text { Dihedral } \\
\text { angle } \\
\text { between the } \\
\text { two rings } \\
\text { (deg) }\end{array}$ & 8.0 & 10.5 & 11.80 & $\begin{array}{l}\text { Dihedral } \\
\text { angle } \\
\text { between the } \\
\text { two rings } \\
\text { (deg) }\end{array}$ & $6.4-11.8$ & 13.64 \\
\hline $\begin{array}{c}U_{\text {eff }}(\mathrm{K}) \\
\text { under zero } \\
\text { dc field }\end{array}$ & 197,323 & 358 & 157 & $\begin{array}{c}U_{\text {eff }}(\mathrm{K}) \\
\text { under zero } \\
\text { dc field }\end{array}$ & none & 141 \\
\hline
\end{tabular}




\section{NMR Spectra}

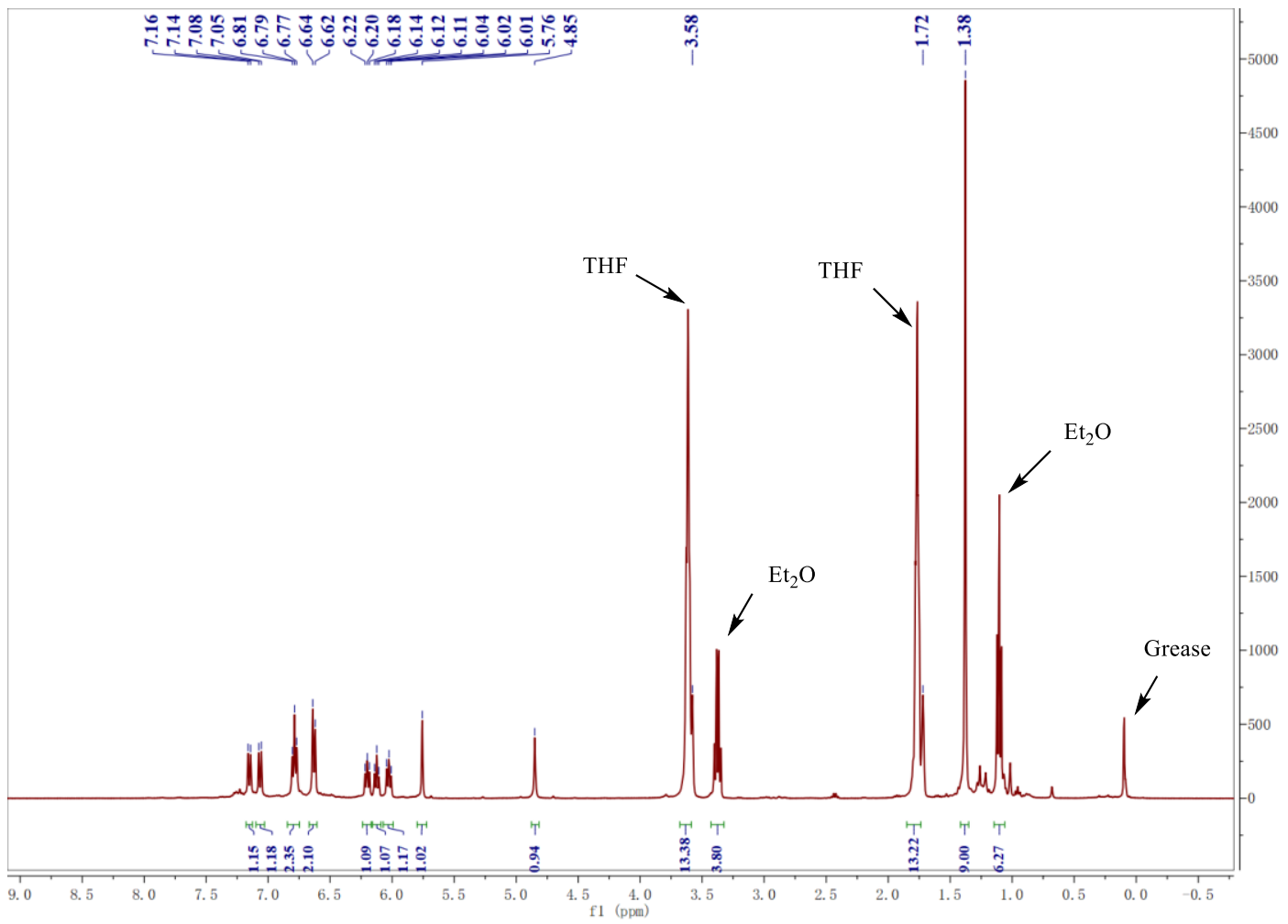

Figure S15. ${ }^{1} \mathrm{H}$ NMR spectrum of benzoborole yttrium chloride in $\mathrm{D}_{8}$-THF.

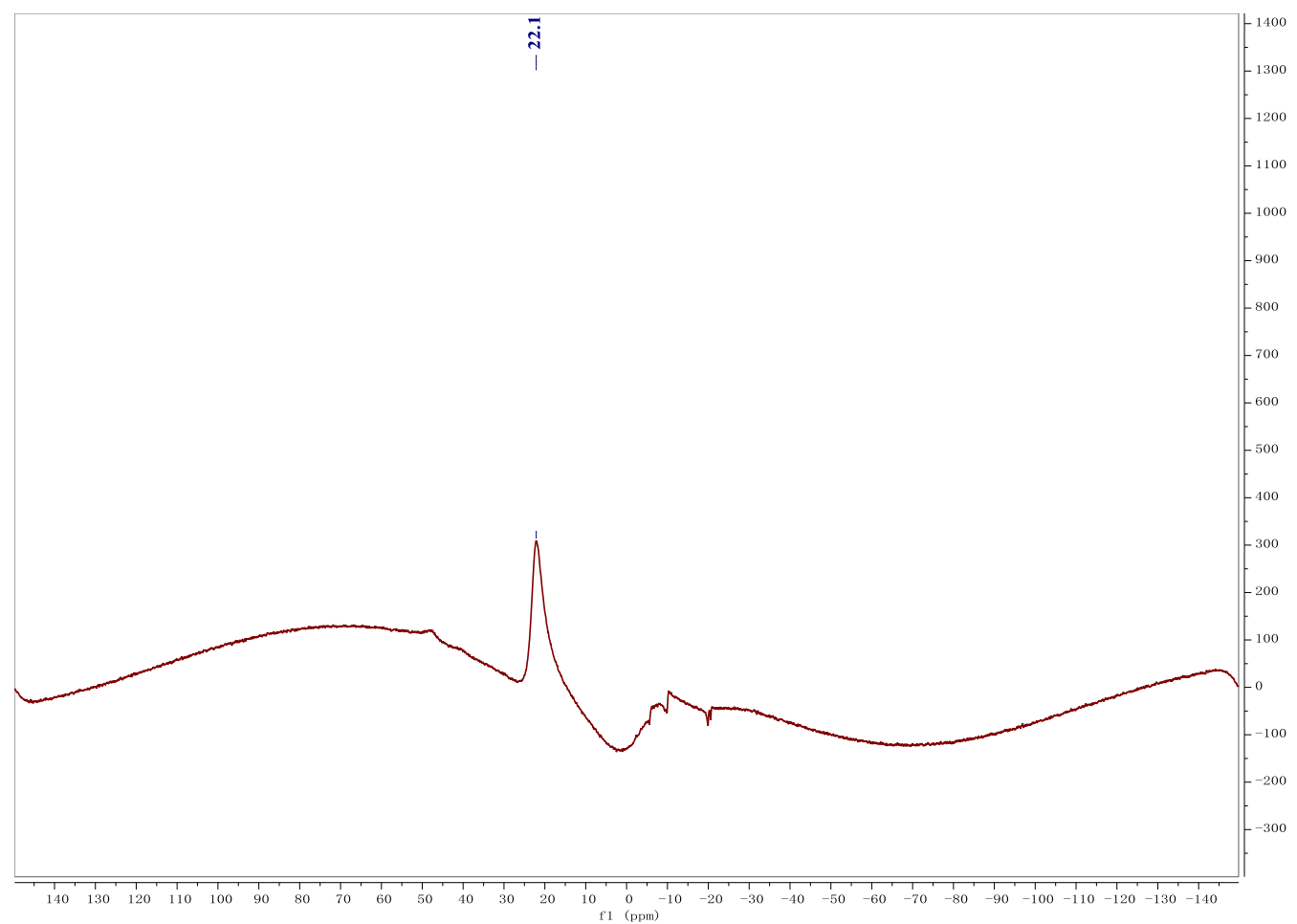

Figure S16. ${ }^{11} \mathrm{~B}$ NMR spectrum of benzoborole yttrium chloride in $\mathrm{D}_{8}$-THF. 


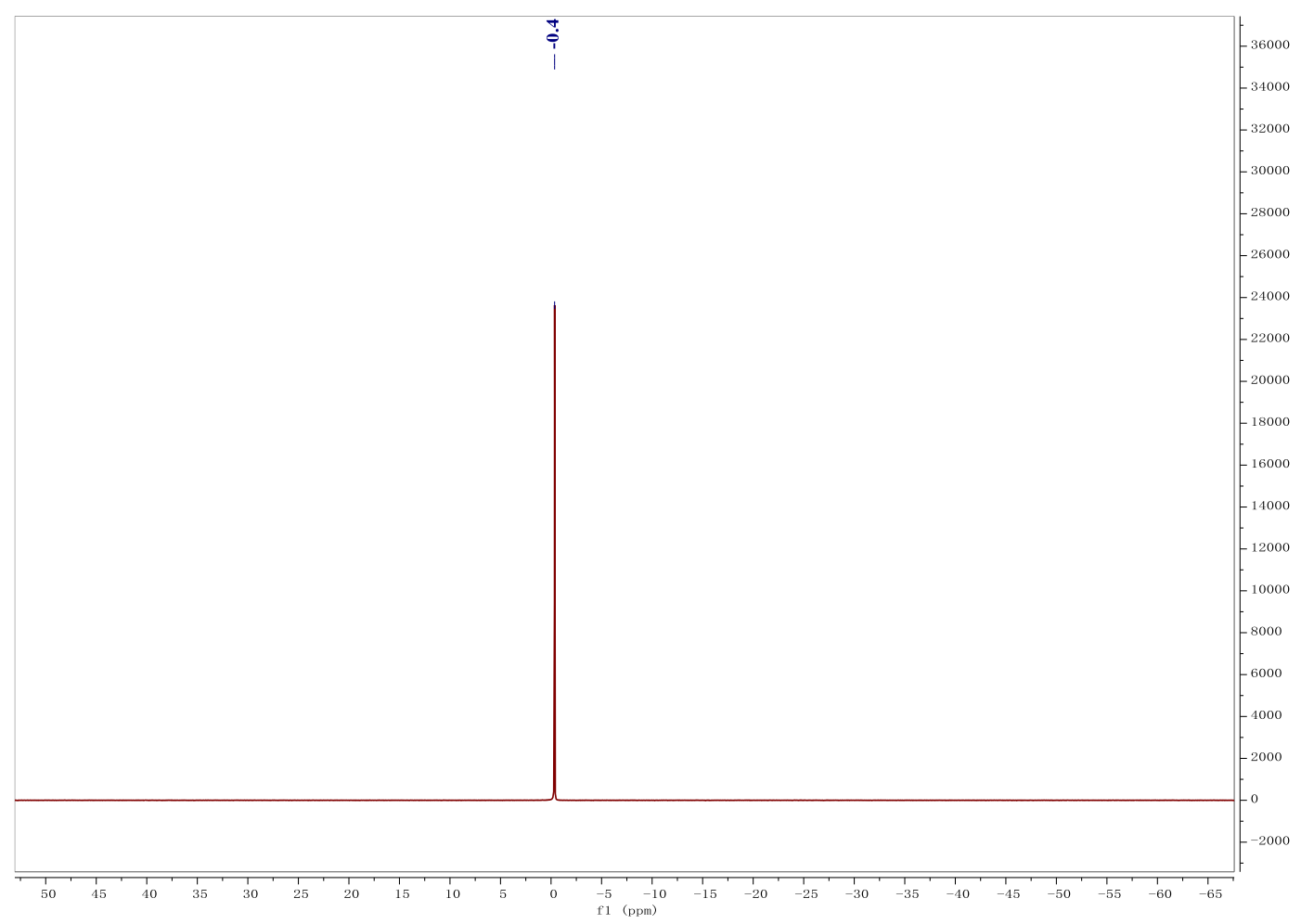

Figure S17. ${ }^{7} \mathrm{Li}$ NMR spectrum of benzoborole yttrium chloride in $\mathrm{D}_{8}$-THF.

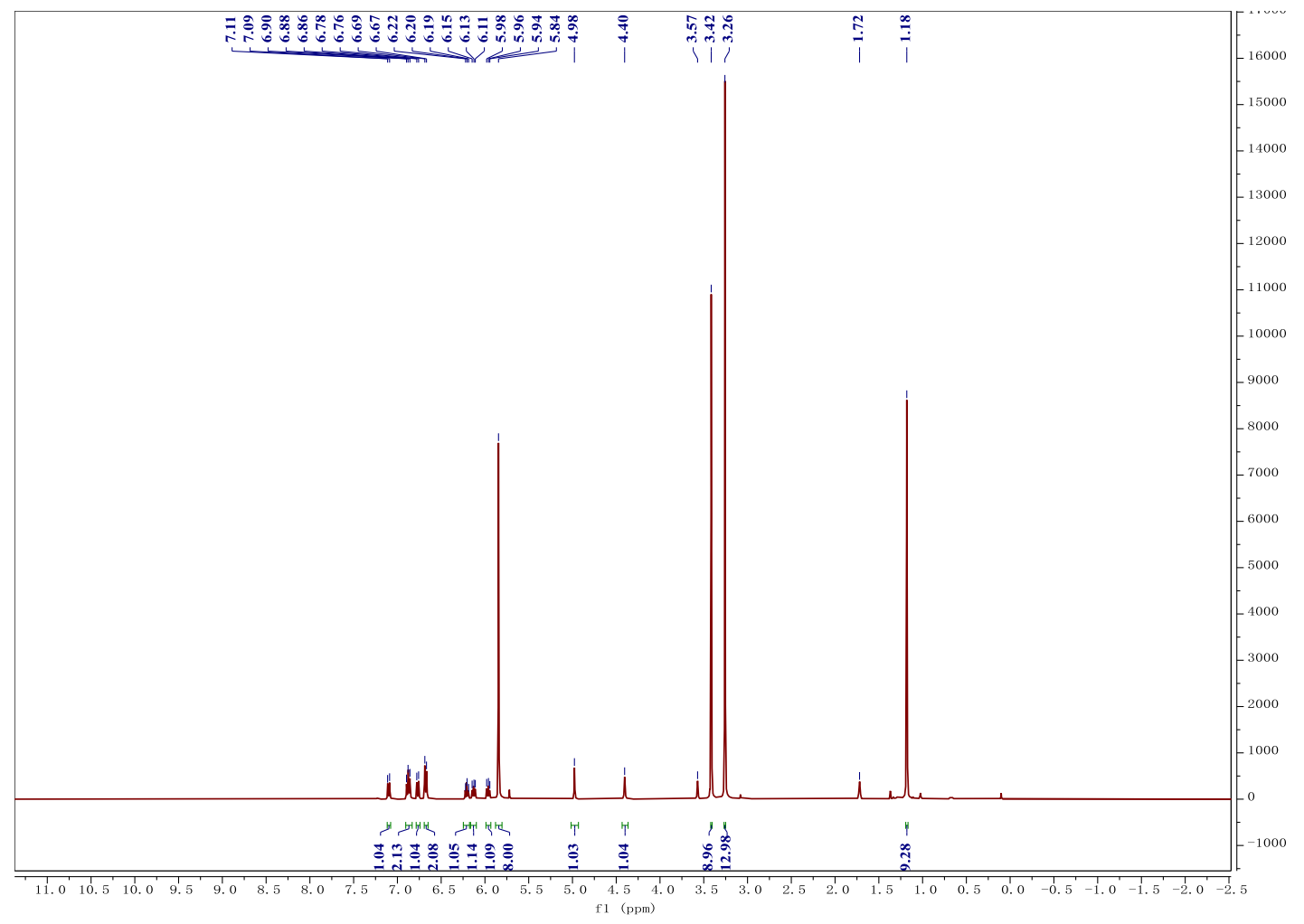

Figure S18. ${ }^{1} \mathrm{H}$ NMR spectrum of $\mathbf{3 Y}$ in $\mathrm{D}_{8}$-THF. 


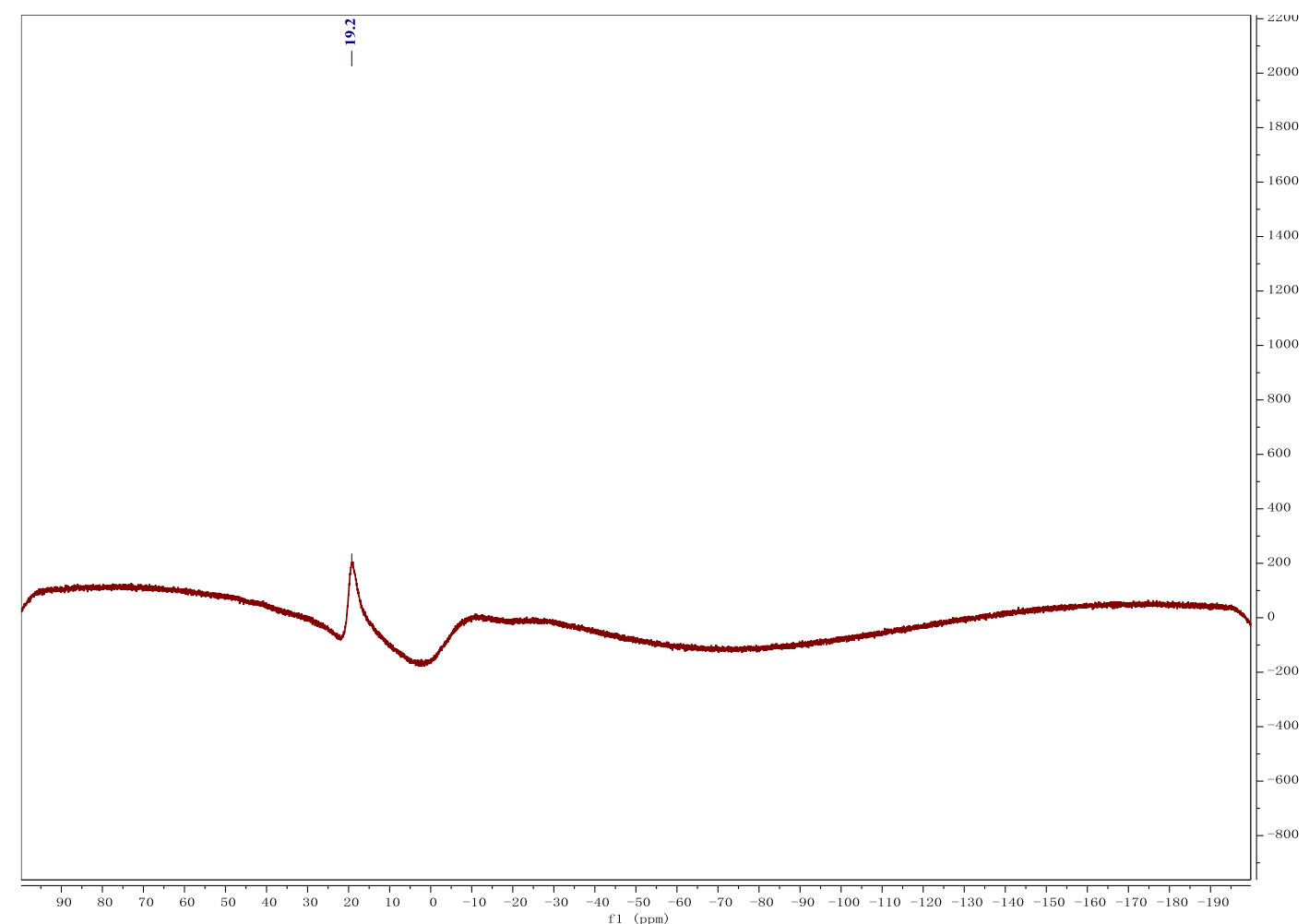

Figure S19. ${ }^{11} \mathrm{~B}$ NMR spectrum of $\mathbf{3 Y}$ in $\mathrm{D}_{8}$-THF.

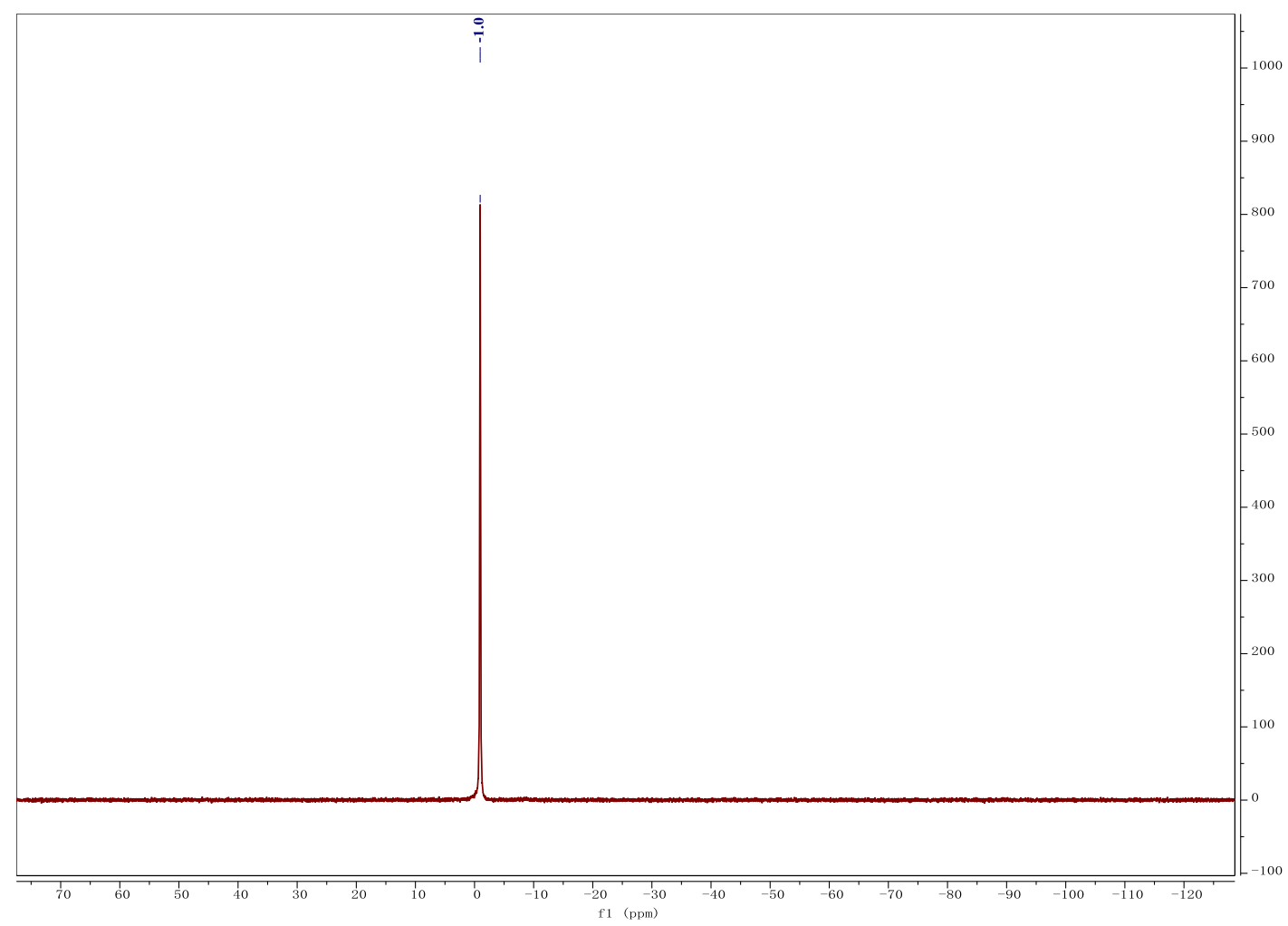

Figure S20. ${ }^{7} \mathrm{Li}$ NMR spectrum of $\mathbf{3 Y}$ in $\mathrm{D}_{8}$-THF. 


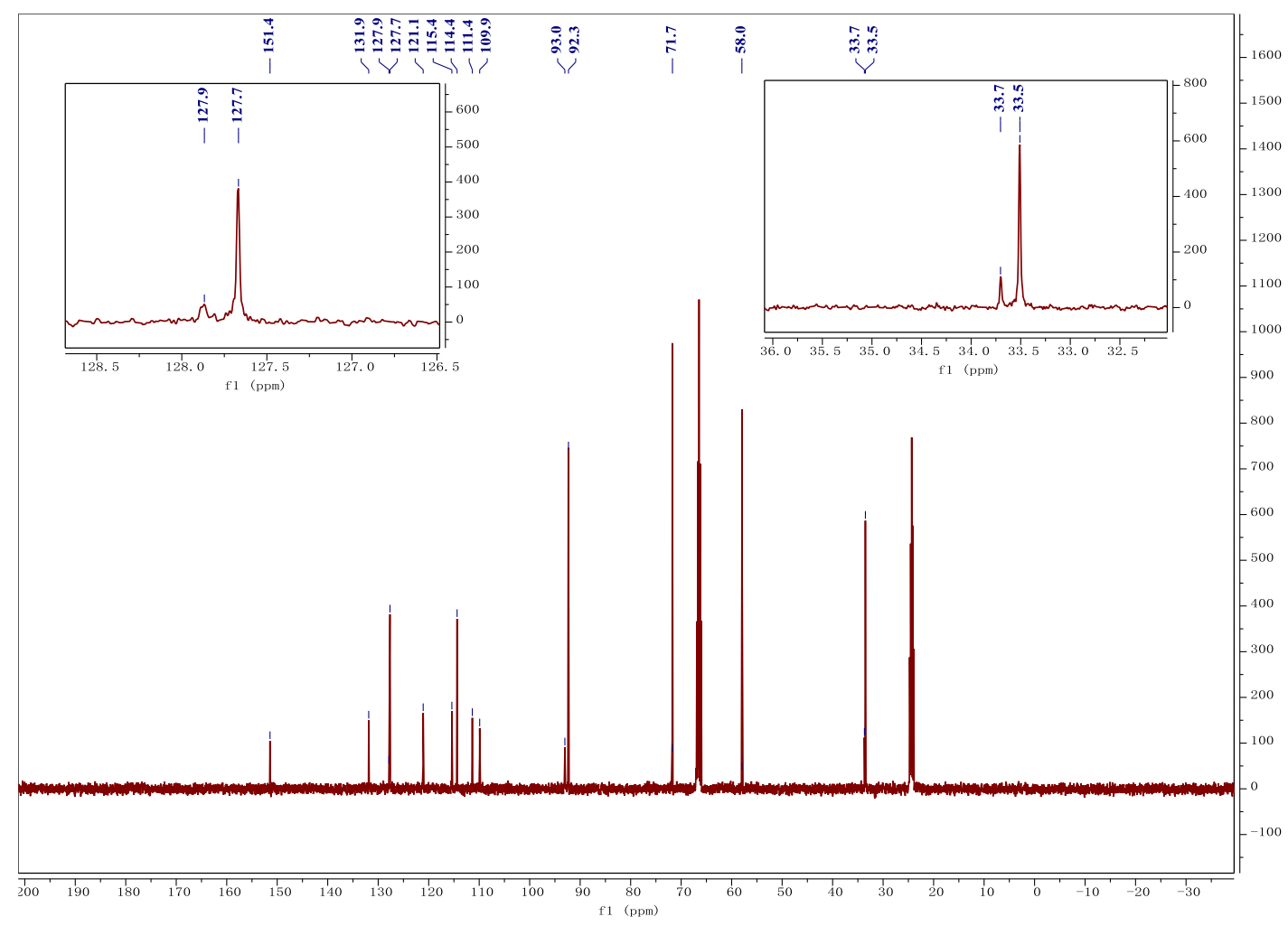

Figure S21. ${ }^{13} \mathrm{C}$ NMR spectrum of $\mathbf{3 Y}$ in $\mathrm{D}_{8}$-THF 


\section{IR Spectra}

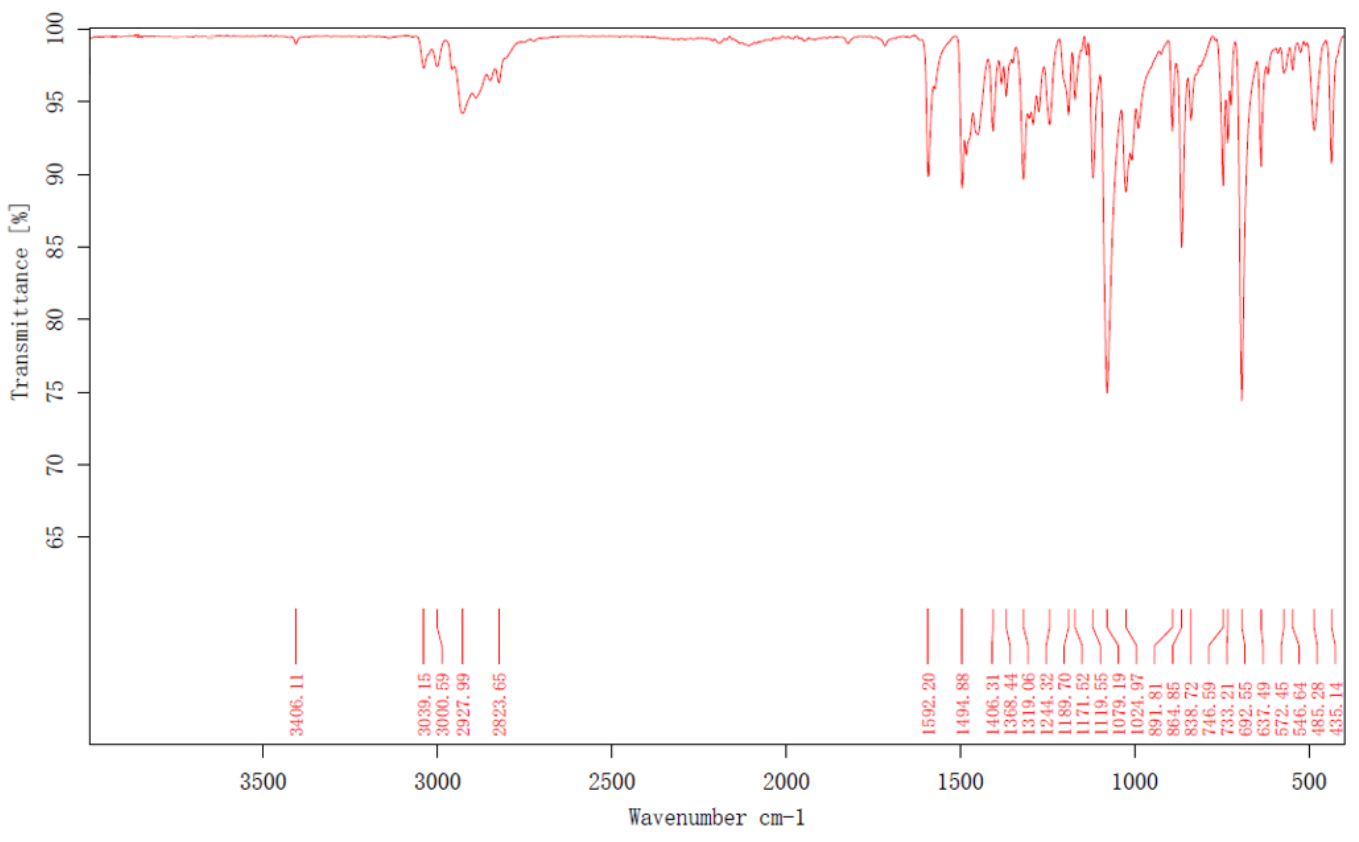

Figure S22. IR spectrum of $\mathbf{3 Y}$ in the solid state under argon atmosphere.

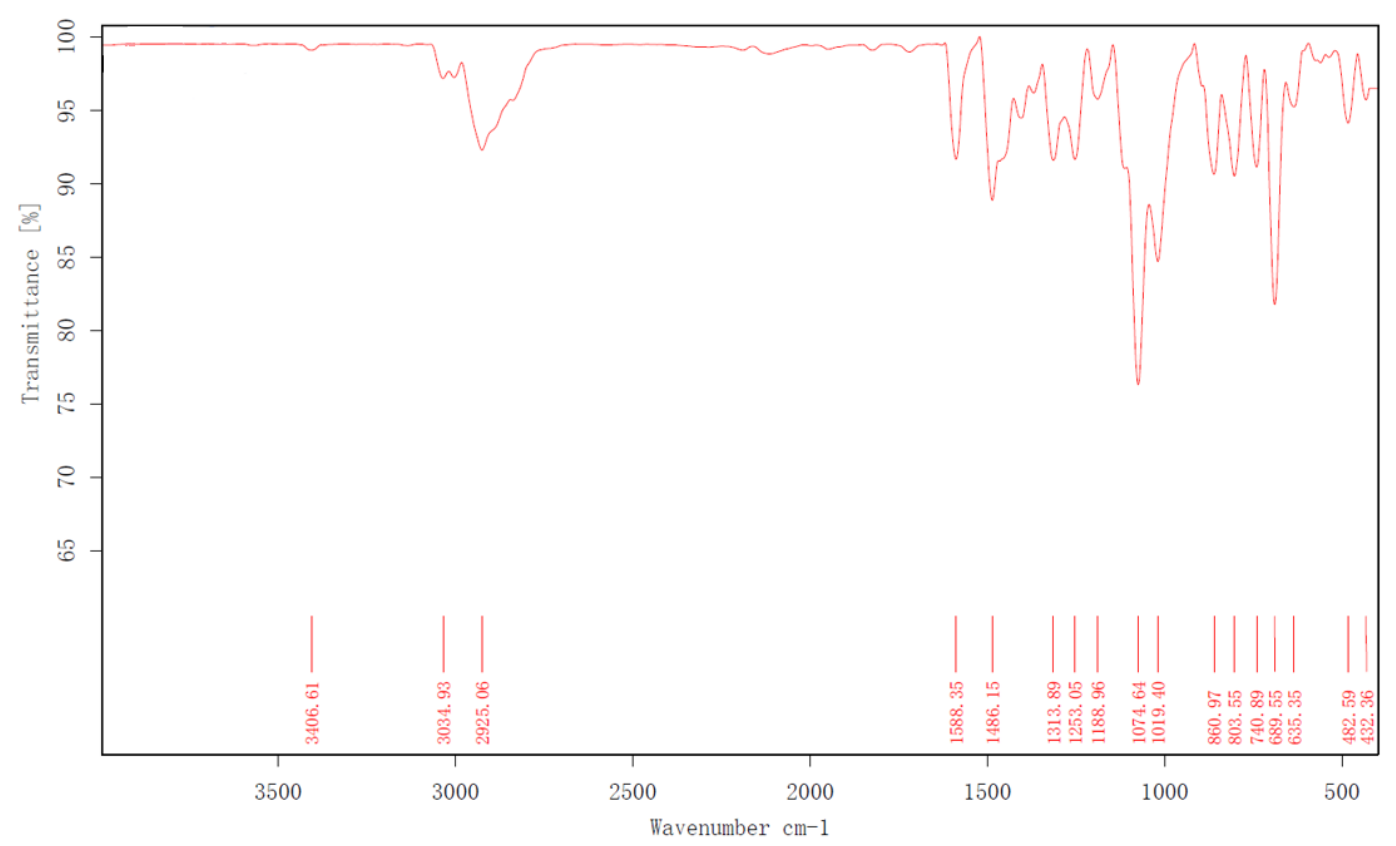

Figure S23. IR spectrum of 3Dy in the solid state under argon atmosphere. 


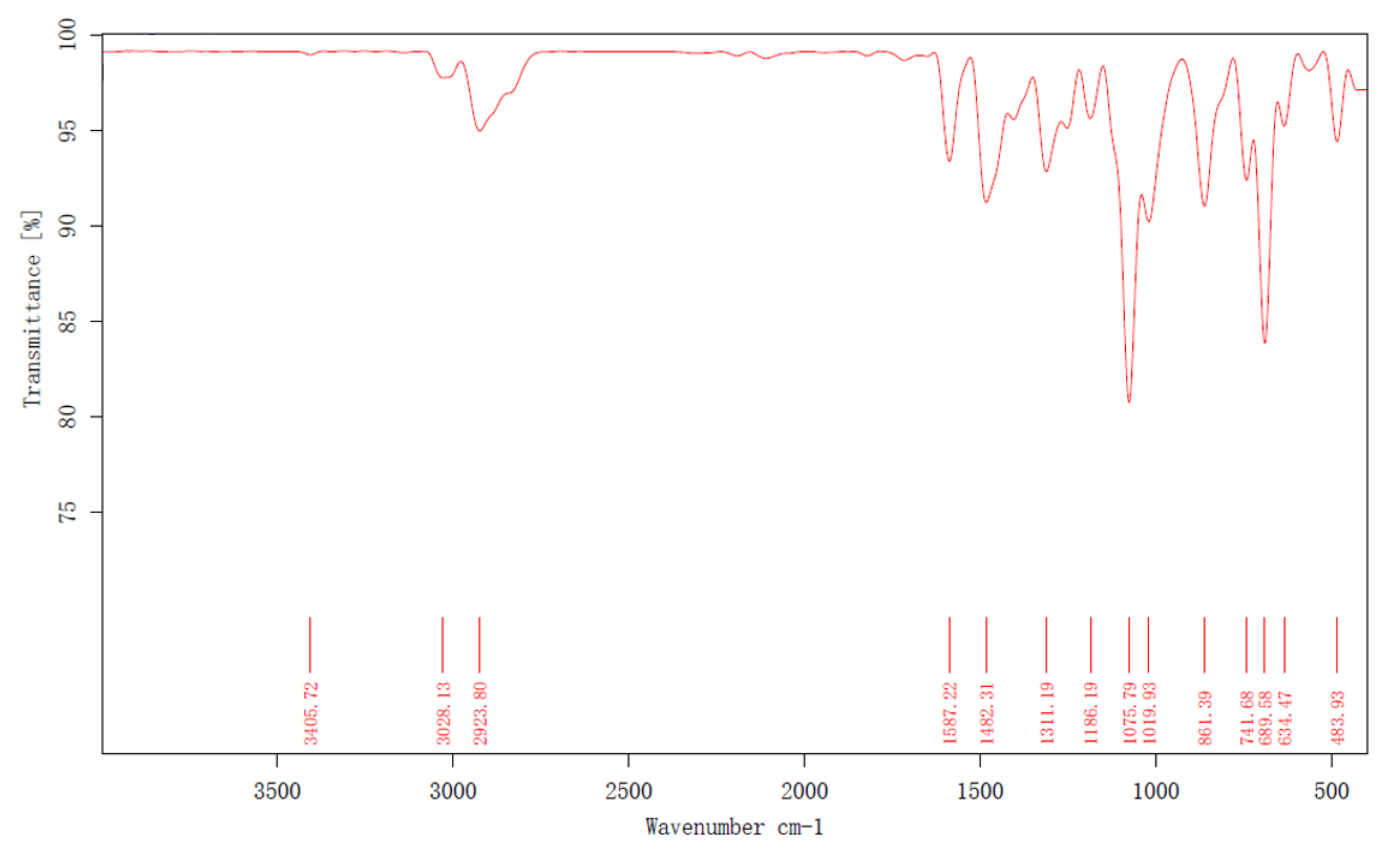

Figure S24. IR spectrum of 3Er in the solid state under argon atmosphere. 


\section{$6 \mathrm{UV}$-vis spectra}

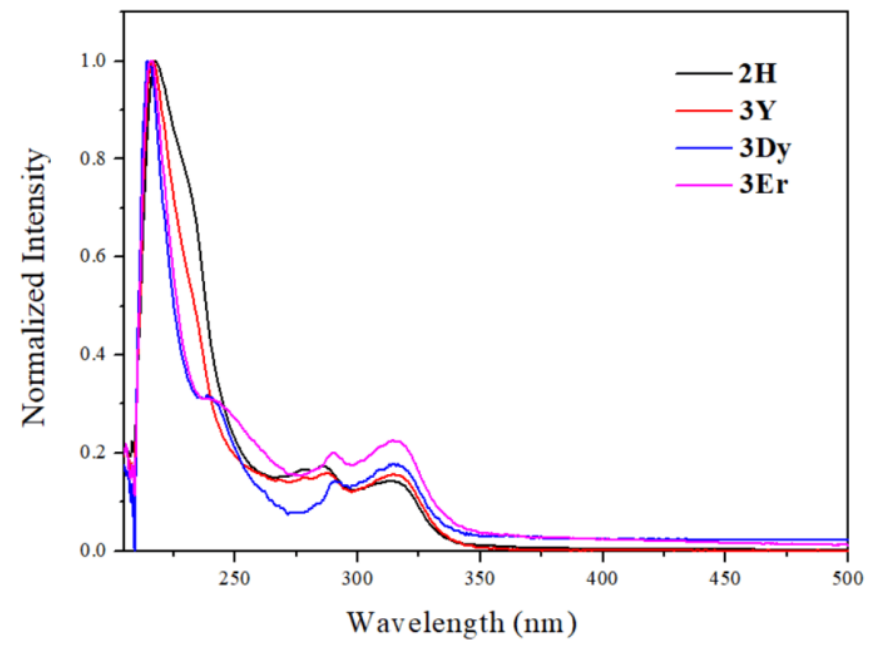

Figure S25. UV-vis absorption spectra of $\mathbf{2 H}, \mathbf{3 Y}, \mathbf{3 D y}$ and $\mathbf{3 E r}$ in THF solution at $10^{-6} \mathrm{M}$. 


\section{Reference}

[S1] Zhu, D.; Guo, L.; Li, J.; Cui, C. From BN Naphthalenes to Benzoborole Dianions. Chem. - Eur. J. 2021, 27, 9514-9518.

[S2] Hu, N.; Gong, L.; Jin, Z.; Chen, W. Crystal Structure of Cyclooctatetraenylpotassium, $\mathrm{C}_{8} \mathrm{H}_{8} \mathrm{~K}_{2} \cdot\left(\mathrm{OC}_{4} \mathrm{H}_{8}\right)_{3}$. J. Organomet. Chem. 1988, 352, 61-66.

[S3] Dolomanov, O. V.; Bourhis, L. J.; Gildea, R. J.; Howard, J. A. K.; Puschmann, H. OLEX2: A Complete Structure Solution, Refinement and Analysis Program. J. Appl. Cryst. 2009, 42, 339-341. [S4] Sheldrick, G. M. SHELXS-90/96, Program for Structure Solution, Acta Crystallogr. Sect. A 1990, 46,467 .

[S5] Sheldrick, G. M. SHELXL 97, Program for Crystal Structure Refinement, University of Goettingen, Goettingen, Germany, 1997. 